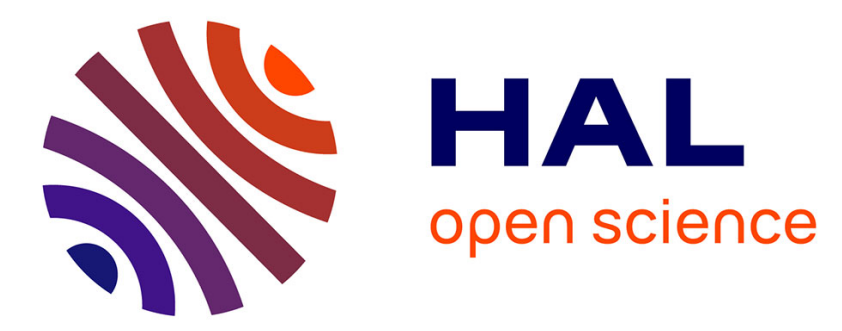

\title{
Etude par analyse enthalpique différentielle d'un verre métallique Fe-Cr-C-P-Si. Influence de la vitesse d'hypertrempe
}

\author{
F. Fouquet, J.P. Allemand, J. Perez, B. de Guillebon
}

\section{- To cite this version:}

F. Fouquet, J.P. Allemand, J. Perez, B. de Guillebon. Etude par analyse enthalpique différentielle d'un verre métallique Fe-Cr-C-P-Si. Influence de la vitesse d'hypertrempe. Revue de Physique Appliquée, 1987, 22 (6), pp.389-397. 10.1051/rphysap:01987002206038900 . jpa-00245553

HAL Id: jpa-00245553

https://hal.science/jpa-00245553

Submitted on 1 Jan 1987

HAL is a multi-disciplinary open access archive for the deposit and dissemination of scientific research documents, whether they are published or not. The documents may come from teaching and research institutions in France or abroad, or from public or private research centers.
L'archive ouverte pluridisciplinaire HAL, est destinée au dépôt et à la diffusion de documents scientifiques de niveau recherche, publiés ou non, émanant des établissements d'enseignement et de recherche français ou étrangers, des laboratoires publics ou privés. 


\title{
Etude par analyse enthalpique différentielle d'un verre métallique Fe-Cr-C-P-Si. Influence de la vitesse d'hypertrempe
}

\author{
F. Fouquet $\left({ }^{*}\right)$, J. P. Allemand $(*)$, J. Perez $\left({ }^{*}\right)$ et B. de Guillebon $\left({ }^{* *}\right)$ \\ (*) Groupe d'Etudes de Métallurgie Physique et de Physique des Matériaux \\ U.A. C.N.R.S. 341, Bât. 502, I.N.S.A., 69621 Villeurbanne Cedex, France \\ (**) Centre de Recherches de Pont-à-Mousson, B.P. 28, 54703 Pont-à-Mousson, France
}

(Reçu le 27 octobre 1986, accepté le 2 mars 1987)

\begin{abstract}
Résumé. - Les rubans de verre métallique ont été obtenus par hypertrempe du liquide avec différentes vitesses du substrat rotatif donc différentes vitesses d'hypertrempe. L'influence de cette vitesse sur l'état structural des rubans bruts d'élaboration mais également sur la relaxation structurale ultérieure a été précisée en utilisant l'analyse enthalpique différentielle; outre les informations concernant les effets thermiques associés à la relaxation ou à la cristallisation des différents rubans, cette technique nous a permis de déterminer la température de Curie et de suivre son évolution avec les traitements thermiques. Les résultats obtenus ont été discutés en liaison avec l'évolution des propriétés mécaniques, ce qui a amené à conclure que l'influence de la vitesse d'hypertrempe pouvait s'expliquer par la seule évolution structurale à l'état vitreux. En se basant sur la théorie d'Egami et al. reposant sur la notion de défauts dans les amorphes métalliques, une interprétation, conduisant à la décomposition de la relaxation structurale en deux grands stades, a été avancée.
\end{abstract}

\begin{abstract}
A Fe-Cr-C-P-Si metallic glass was produced by melt-spinning technique with various rates of the rotating strip and consequently different quench rates. The influence of this quench rate on the structural state of the as-quenched ribbons and on the subsequent structural relaxation has been studied by mean of differential scanning calorimetry (DSC). Beyond informations about thermal effects related to structural relaxation and crystallization of the various ribbons, DSC experiments have allowed to determine the Curie temperature and to follow its evolution with heat treatments. A comparison has been done with the evolution of the mechanical properties and it should be assumed that the changes of properties as a function of quench rate are due to structural evolution of the glassy state. Using the theory of Egami et al. based on the concept of «defects" in amorphous metals, an interpretation is given which leads to divide the structural relaxation in two main stages.
\end{abstract}

\section{Introduction.}

Pour un alliage vitrifiable de composition donnée, l'état vitreux susceptible d'être obtenu par hypertrempe depuis l'état liquide n'est pas thermodynamiquement défini et n'est pas unique. L'alliage à l'état vitreux peut ainsi se présenter dans des configurations structurales différentes selon les conditions d'élaboration et en particulier selon la vitese d'hypertrempe qui détermine la température à laquelle le liquide est figé à l'état de verre. Par ailleurs, pour des conditions d'élaboration données, il se produira lors d'un recuit ultérieur du verre des modifications configurationnelles au sein de l'alliage amorphe, phénomène connu sous le nom de relaxation structurale. De nombreuses propriétés physiques et mécani- ques sont sensibles à cette relaxation structurale et il est raisonnable de penser qu'elles doivent l'être également au changement des conditions d'élaboration. Cela pourrait d'ailleurs être à l'origine des désaccords entre les résultats obtenus par divers auteurs pour un même alliage. En fait, relativement peu de travaux ont porté sur l'influence des conditions d'élaboration ; ceci est dû pour une large part à la difficulté de contrôler la variation des paramètres de production des rubans tout en obtenant un alliage amorphe.

Les travaux réalisés dans ce domaine [1-9] portent tous sur des rubans de verres métalliques obtenus par hypertrempe sur roue et le principal paramètre étudié est la vitesse de rotation de la roue dont dépendent la vitesse d'hypertrempe et l'épaisseur 
des rubans. Les propriétés magnétiques [1-5] et mécaniques [6-9] ont été le plus fréquemment utilisées pour mettre en évidence l'influence de cette vitesse sur l'état structural des verres métalliques obtenus. Les différents auteurs ont cherché non seulement à caractériser les rubans bruts d'élaboration mais également à préciser l'évolution des propriétés en relation avec la relaxation structurale. A cet effet, la température de Curie $\left(T_{\mathrm{C}}\right)$, grandeur particulièrement sensible à cette relaxation structurale [10-11], a été largement utilisée $[1,3,5,6]$ dans le cas des verres ferromagnétiques. Les résultats obtenus par les auteurs ne sont pas toujours concordants, Mizoguchi et al. [3] et Greer [5] observent ainsi une variation sensible de $T_{\mathrm{C}}$ avec la vitesse d'élaboration alors que Takayama et Oi [1] n'en observent pas.

Pour notre part, nous avons effectué récemment un travail concernant l'influence de la vitesse d'élaboration sur diverses propriétés d'un alliage FeCr-C-P-Si [12]. L'étude des propriétés mécaniques et micromécaniques nous a en particulier permis de mettre en évidence une augmentation du module d'Young et une diminution de la fragilité des rubans quand cette vitesse augmente [12,13], rejoignant ainsi les résultats de Chen et al. [7] et de Gerling et Wagner [6].

Dans le présent article, nous nous sommes attachés, pour ce même alliage et les mêmes conditions d'élaboration à préciser comment la vitesse d'hypertrempe affecte l'état et la stabilité structurale des verres métalliques obtenus en utilisant l'analyse enthalpique différentielle; cette technique d'étude nous permettant entre autres de suivre l'évolution des températures de cristallisation ou de Curie avec l'état structural. Nous discuterons les résultats obtenus en regard de l'évolution des propriétés mécaniques étudiée par ailleurs $[12,13]$.

\section{Méthode expérimentale.}

Les rubans étudiés de composition $\mathrm{Fe}_{75} \mathrm{Cr}_{4} \mathrm{C}_{10,5} \mathrm{P}_{8,5} \mathrm{Si}_{2}$ ont été élaborés par hypertrempe du liquide sur une bande d'acier entraînée en rotation. A débit de métal liquide constant, une vitesse de défilement de la bande élevée conduit à une épaisseur de ruban faible et donc à une vitesse de refroidissement élevée. L'épaisseur $e$ du ruban et la vitesse $V$ de défilement de la bande sont reliées par une relation du type $e \simeq V^{-a}$ avec $a \simeq 0,75$. Les contrôles effectués en diffraction $\mathrm{X}$ sur les deux faces du ruban ont montré qu'ils étaient parfaitement amorphes pour des vitesses $V$ comprises entre 20 et $46 \mathrm{~m} / \mathrm{s}$. Ces deux valeurs extrêmes correspondent respectivement à des vitesses d'hypertrempe de 4 et $8 \times 10^{5}{ }^{\circ} \mathrm{C} / \mathrm{s}$, vitesses évaluées - à l'aide d'un modèle thermique - en sortie de la flaque liquide formée lors de l'impact du jet sur le substrat rotatif

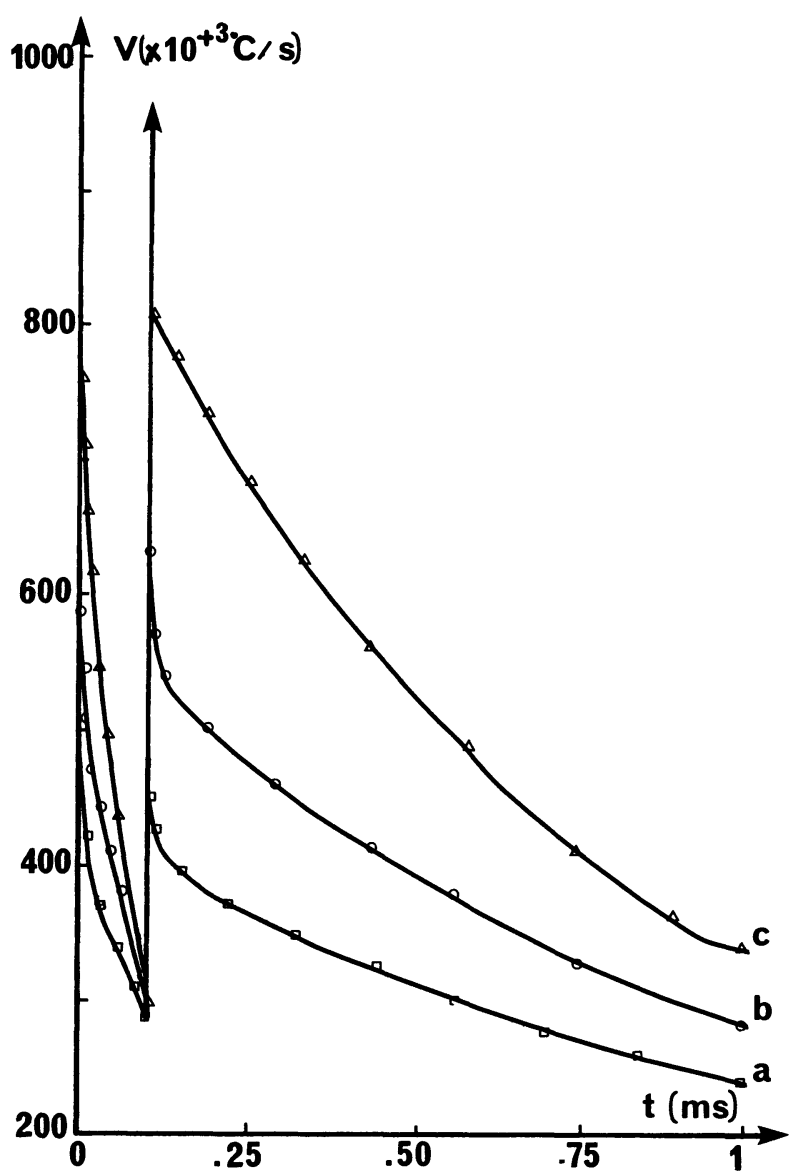

Fig. 1. - Evolution de la vitesse de refroidissement au cours du temps pour différentes vitesses linéaires du substrat rotatif $(V=20 \mathrm{~m} / \mathrm{s}$ (a) $; 35 \mathrm{~m} / \mathrm{s} \mathrm{(b)} ; 50 \mathrm{~m} / \mathrm{s}$ (c)).

[Cooling rate versus time for various rates of the rotating strip. $(V=20 \mathrm{~m} / \mathrm{s}$ (a) $; 35 \mathrm{~m} / \mathrm{s}$ (b) $; 50 \mathrm{~m} / \mathrm{s}$ (c)).]

que constitue la bande d'acier (Fig. 1). L'épaisseur moyenne des rubans passe dans le même temps de 37 à $23 \mu \mathrm{m}$; les valeurs de cette épaisseur pour les six vitesses $V$ mises en œuvre sont données dans le tableau I. Par la suite, nous désignerons par $R_{V}$ le ruban élaboré à la vitesse $V$.

\section{Tableau I.}

$\begin{array}{lllllll}V(\mathrm{~m} / \mathrm{s}) & 20 & 27 & 33 & 36 & 42 & 46 \\ e(\mu \mathrm{m}) & 37 & 31 & 27,5 & 26,5 & 25,5 & 23\end{array}$

L'analyse enthalpique différentielle (AED) a été réalisée à l'aide d'un analyseur thermique Dupont 990 équipé d'une cellule DSC-910. Les mesures ont été effectuées entre 20 et $600^{\circ} \mathrm{C}$ avec des vitesses de balayage en température comprises entre 0,5 et $10^{\circ} \mathrm{C} / \mathrm{min}$ sous atmosphère d'argon purifié.

Les traitements thermiques réalisés sur les rubans ont été effectués soit in situ dans l'analyseur thermique soit sous vide primaire ou atmosphère neutre 
(argon) dans une ampoule de pyrex scellée plongée dans un bain de sel.

\section{Résultats expérimentaux. Discussion.}

Nous présentons d'abord les résultats obtenus pour le ruban $R_{33}$ puis nous comparerons ensuite avec les autres rubans.

\subsection{THERMOGRAMME OBTENU A PARTIR D'UN ÉTAT} BRUT D'HYPERTREMPE. - La figure 2 montre le thermogramme obtenu pour un échantillon du ruban $R_{33}$ avec une vitesse de balayage en température de $5^{\circ} \mathrm{C} / \mathrm{min}$. On note à partir de $200^{\circ} \mathrm{C}$ un léger effet

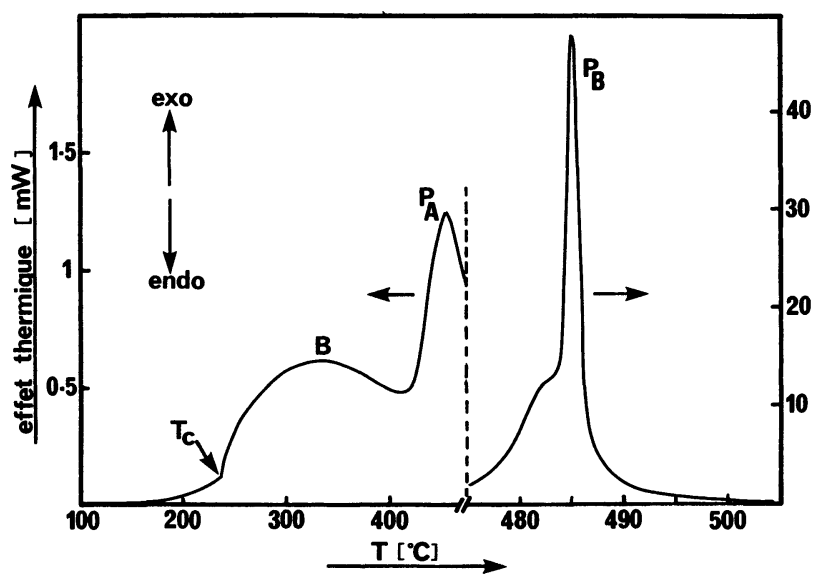

Fig. 2. - Thermogramme d'un échantillon du ruban $R_{33}$ brut d'hypertrempe. Balayage en température à $5{ }^{\circ} \mathrm{C} / \mathrm{min}$.

[Thermogram for a sample of the as-quenched $R_{33}$ ribbon (scanning rate $=5^{\circ} \mathrm{C} / \mathrm{min}$ ).

exothermique qui correspond au début de la relaxation structurale. A $236^{\circ} \mathrm{C}$, on observe un brusque changement de pente qui correspond à la température de Curie $T_{\mathrm{C}}$ de l'alliage, la transition ferroparamagnétique s'accompagnant d'une brutale variation de $C_{\mathrm{p}}$; ce phénomène se superpose à l'effet exothermique associé à la relaxation structurale qui conduit à la bosse $\mathrm{B}$ sur le thermogramme. La cristallisation apparaît au-delà de $400^{\circ} \mathrm{C}$ et se traduit par l'existence de deux pics exothermiques $\mathrm{P}_{\mathrm{A}}\left(454^{\circ} \mathrm{C}\right)$ et $P_{B}\left(485^{\circ} \mathrm{C}\right)$. Le pic $P_{A}$ de beaucoup plus faible amplitude que $\mathrm{P}_{\mathrm{B}}$ correspond à une cristallisation primaire de fer $\alpha$ ou tout au moins d'une solution solide, base fer de structure cubique centrée. Le pic $\mathrm{P}_{\mathrm{B}}$ qui présente un épaulement du côté basse température semble résulter de la cristallisation de plusieurs phases au sein de la matrice amorphe, phases complexes difficiles à identifier en diffraction $\mathbf{X}$.

3.2 EVOLUTION DES THERMOGRAMMES AVEC L'ÉTAT STRUCTURAL DE DÉPART. - Des balayages en température (à $5^{\circ} \mathrm{C} / \mathrm{min}$ ) successifs, chacun suivi d'un refroidissement rapide ont été effectués sur le ruban $R_{33}$ (Fig. 3). La montée (1) jusqu'à $400^{\circ} \mathrm{C}$, caractérise l'évolution structurale de l'alliage sans cristallisation ; la seconde montée (2) jusqu'à $470^{\circ} \mathrm{C}$ caractérise le matériau relaxé lors de la montée (1) et recouvre la cristallisation primaire $\left(\mathrm{P}_{\mathrm{A}}\right)$; la mon-

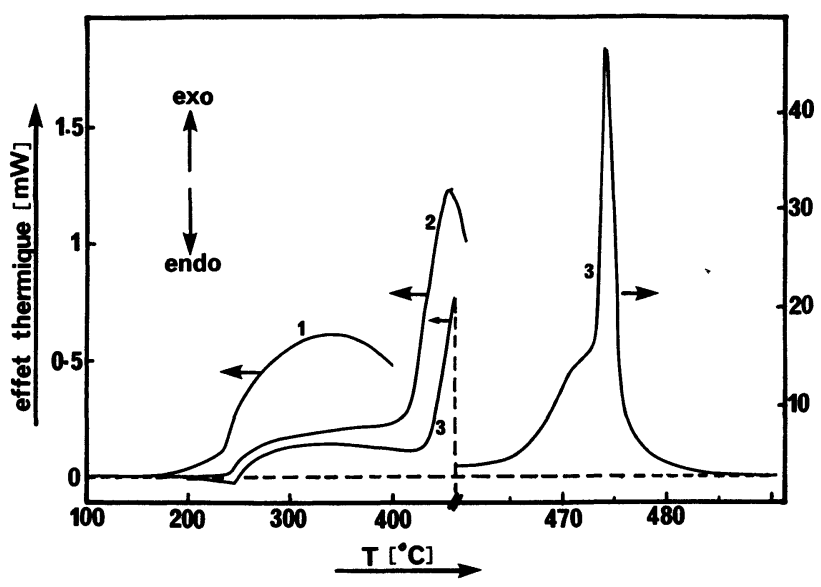

Fig. 3. - Thermogrammes d'un échantillon du ruban $R_{33}$. Montées successives en température à $5^{\circ} \mathrm{C} / \mathrm{min}$ suivies d'un refroidissement rapide : (1) montée jusqu'à $400^{\circ} \mathrm{C}$; (2) montée jusqu'à $460^{\circ} \mathrm{C}$; (3) montée jusqu'à $500^{\circ} \mathrm{C}$.

[Thermograms for a sample of the $R_{33}$ ribbon. Successive scannings at $5{ }^{\circ} \mathrm{C} / \mathrm{min}$ followed by rapid cooling : (1) scanning up to $400^{\circ} \mathrm{C}$; (2) scanning up to $460^{\circ} \mathrm{C}$; (3) scanning up to $500^{\circ} \mathrm{C}$.]

tée (3) jusqu'à $590^{\circ} \mathrm{C}$ permet d'obtenir la cristallisation totale de l'alliage $\left(\mathrm{P}_{\mathrm{B}}\right)$, consécutivement à la cristallisation primaire produite lors de la montée (2). La comparaison des thermogrammes (1), (2) et (3) de la figure 3 et de celui de la figure 2 permet de constater que :

- la température de Curie augmente de $9^{\circ} \mathrm{C}$ entre les montées [1] et [3] et passe ainsi de 236 à $245^{\circ} \mathrm{C}$. L'anomalie au passage de $T_{\mathrm{C}}$ est très nettement visible lors de la montée [3], ceci indique que le verre métallique est encore en grande partie amorphe après montée à $470^{\circ} \mathrm{C}$; la cristallisation primaire à l'origine du pic $P_{A}$ ne doit affecter qu'une faible fraction du matériau ;

- l'effet exothermique associé à la relaxation structurale mis en évidence sur le thermogramme (1) disparaît en (2) ;

- la température du pic $P_{A}$ n'est pas affectée par une relaxation structurale préalable ;

- la température du pic $\mathrm{P}_{\mathrm{B}}$ lors de la montée (3) est abaissée de $11^{\circ} \mathrm{C}$ par rapport à une montée directe depuis l'état brut d'élaboration $\left(T_{\mathrm{B}}=474{ }^{\circ} \mathrm{C}\right.$ au lieu de $485^{\circ} \mathrm{C}$ ). Le deuxième stade de cristallisation apparaît donc fortement dépendant du premier. 
3.3 INFLUENCE DE LA VITESSE DE BALAYAGE EN TEMPÉRATURE. - Pour compléter notre étude sur le ruban $R_{33}$ des thermogrammes ont été réalisés avec des vitesses de balayage $\left(V_{B}\right)$ en température de $0,5,1,2$ et $10^{\circ} \mathrm{C}$. Des essais ont également été effectués à $20^{\circ} \mathrm{C} / \mathrm{min}$ mais jusqu'à $300^{\circ} \mathrm{C}$ seulement. Les thermogrammes obtenus qui restent qualitativement les mêmes que pour une vitesse de balayage de $5{ }^{\circ} \mathrm{C} / \mathrm{min}$, ne seront pas présentés. Dans le tableau II ci-dessous sont seulement indiquées les températures $T_{\mathrm{C}}, T_{\mathrm{A}}$ et $T_{\mathrm{B}}$ (voir Fig. 2) correspondant à chaque vitesse.

Tableau II.
$V_{\mathrm{B}}\left({ }^{\circ} \mathrm{C} / \mathrm{mn}\right)$

$T_{\mathrm{C}}\left({ }^{\circ} \mathrm{C}\right)$

$T_{\mathrm{A}}\left({ }^{\circ} \mathrm{C}\right)$

$T_{\mathrm{B}}\left({ }^{\circ} \mathrm{C}\right)$
0,5

238

436

456

1
238
442
464

\section{2}

237

447

475

5
236
454
485

10

236

462

494
20

236

$-$
D'après ce tableau nous remarquons que la température de Curie reste indépendante de $V_{\mathrm{B}}$ pour $V_{\mathrm{B}}>5^{\circ} \mathrm{C} / \mathrm{min}$. Pour ces vitesses la relaxation structurale du verre pendant la montée en température jusqu'à $236^{\circ} \mathrm{C}$ apparaît donc insuffisante pour provoquer une augmentation significative de $T_{\mathrm{C}}$. L'effet de cette relaxation structurale sur $T_{\mathrm{C}}$ est par contre visible pour les vitesses de balayage inférieures à $5^{\circ} \mathrm{C} / \mathrm{min}$. L'augmentation de $T_{\mathrm{C}}$ observée $\left(2^{\circ} \mathrm{C}\right)$ est cependant faible ce qui montre qu'en dessous de $240^{\circ} \mathrm{C}$ la relaxation structurale reste limitée. En outre on obtient la même valeur de $T_{\mathrm{C}}$ pour $V_{\mathrm{B}}=1{ }^{\circ} \mathrm{C} / \mathrm{min}$ et $V_{\mathrm{B}}=0,5^{\circ} \mathrm{C} / \mathrm{min}$. Ceci semble par contre indiquer que l'évolution structurale produite au cours d'une montée en température à $1^{\circ} \mathrm{C} / \mathrm{min}$ est déjà telle que la cinétique de relaxation est très fortement ralentie ; le passage à une vitesse moitié ne peut alors entraîner qu'une faible différence d'état structural non décelable au niveau de $T_{\mathrm{C}}$.

Les énergies d'activation apparentes correspondant aux deux stades de la cristallisation ont été déterminées à partir des données du tableau II par la méthode de Kissinger-Boswell [14]. Les valeurs trouvées sont $E_{\mathrm{A}} \simeq 3,7 \mathrm{eV}$ pour la cristallisation primaire (pic $\mathrm{P}_{\mathrm{A}}$ ) et $E_{\mathrm{B}} \simeq 2,8 \mathrm{eV}$ pour le deuxième stade de cristallisation (pic $P_{B}$ ). Il est à remarquer qu'au pic $P_{A}$ correspond une énergie d'activation plus élevée qu'au pic $P_{B}$ pourtant situé à plus haute température. De ce fait, on est amené à admettre que la cristallisation correspondante $\left(P_{B}\right)$ ne peut pas se produire sans une cristallisation primaire préalable $\left(\mathrm{P}_{\mathrm{A}}\right)$. Ces cristallites primaires peuvent soit jouer le rôle de sites préférentiels de germination pour la cristallisation de la matrice amorphe, soit induire localement des fluctuations de composition au sein de cette matrice favorisant la dévitrification. Notons toutefois que les valeurs de $E_{\mathrm{A}}$ et surtout de $E_{\mathrm{B}}$, déterminées par la méthode de Kissinger, peu- vent être entachées d'une certaine erreur, le maximum des pics $P_{A}$ et $P_{B}$ ne correspondant pas forcément avec le maximum de vitesse de réaction de chacun des processus, du fait de leur interférence.

3.4 INFLUENCE DE LA VITESSE D'HYPERTREMPE. Des analyses thermiques ont été effectuées sur les rubans $\mathbf{R}_{20}, \mathbf{R}_{27}, \mathbf{R}_{36}, \mathbf{R}_{42}$ et $\mathbf{R}_{46}$ avec une vitesse de balayage de $5^{\circ} \mathrm{C} / \mathrm{min}$. Les thermogrammes apparaissent quasi identiques à celui de la figure 2 quelle que soit la vitesse d'élaboration des rubans entre 20 et $46 \mathrm{~m} / \mathrm{s}$. Aucune différence significative n'est observée au niveau de la température de Curie $T_{\mathrm{C}}$ et des températures des pics de cristallisation $\left(T_{\mathrm{A}}\right.$ et $T_{\mathrm{B}}$ ) comme le montre le tableau III.

Tableau III.

$V(\mathrm{~m} / \mathrm{s})$

$T_{\mathrm{C}}\left({ }^{\circ} \mathrm{C}\right)$

$T_{\mathrm{A}}\left({ }^{\circ} \mathrm{C}\right)$

$T_{\text {B }}\left({ }^{\circ} \mathrm{C}\right)$

\begin{tabular}{rlrllr}
20 & 27 & 33 & \multicolumn{1}{c}{36} & 42 & 46 \\
236 & 235,5 & 236 & 235,5 & 235,5 & 236 \\
455 & 454 & 454 & 454 & 454 & 453 \\
485 & 485 & 485 & 485 & 484 & 486
\end{tabular}

Une étude de la reproductibilité des résultats effectuée sur plusieurs échantillons du ruban $R_{33}$ nous a en effet permis de conclure que $T_{\mathrm{C}}=(236 \pm 0,5){ }^{\circ} \mathrm{C}, \quad T_{\mathrm{A}}=(454 \pm 1){ }^{\circ} \mathrm{C}$ et $T_{\mathrm{B}}=$ $(485 \pm 1)^{\circ} \mathrm{C}$. L'enthalpie de cristallisation a été déterminée pour chacun des rubans, elle est comprise entre $-3,5$ et $-4,2 \mathrm{~kJ} /$ mole mais il n'est pas possible d'établir une corrélation entre cette enthalpie et la vitesse d'élaboration du ruban. Les études en fonction de la vitesse de balayage conduisent à des résultats semblables pour tous les rubans. Le fait que les températures de cristallisation sont indépendantes de la vitesse d'hypertrempe même pour les faibles vitesses de balayage milite en faveur d'une absence de cristallisation même pour les faibles vitesses d'élaboration comme le laissaient penser les contrôles effectués en diffraction $\mathrm{X}$; en effet la préexistence de microcristallites au sein de la matrice amorphe (ou même seulement en surface des rubans) devrait au minimum se traduire par un décalage de la température de cristallisation comme le suggèrent les résultats du paragraphe 3.2.

La seule différence sensible et reproductible observée en fonction de la vitesse d'élaboration se situe au niveau de l'effet exothermique associé à la relaxation structurale. Afin de mettre clairement en évidence cette différence nous avons, à partir des thermogrammes des rubans $\mathbf{R}_{20}, \mathbf{R}_{33}$ et $\mathbf{R}_{46}$, tracé les courbes $C_{\mathrm{p}}=f(T)$ : la figure 4 montre ainsi que la diminution de $C_{\mathrm{p}}$ associée à la relaxation structurale est d'autant plus prononcée que la vitesse $V$ est élévée. Ceci traduirait bien des états vitreux de départ différents, cet état vitreux s'éloignant d'autant plus de l'équilibre métastable que $V$ est grande et conduisant ainsi à une variation d'enthalpie plus 


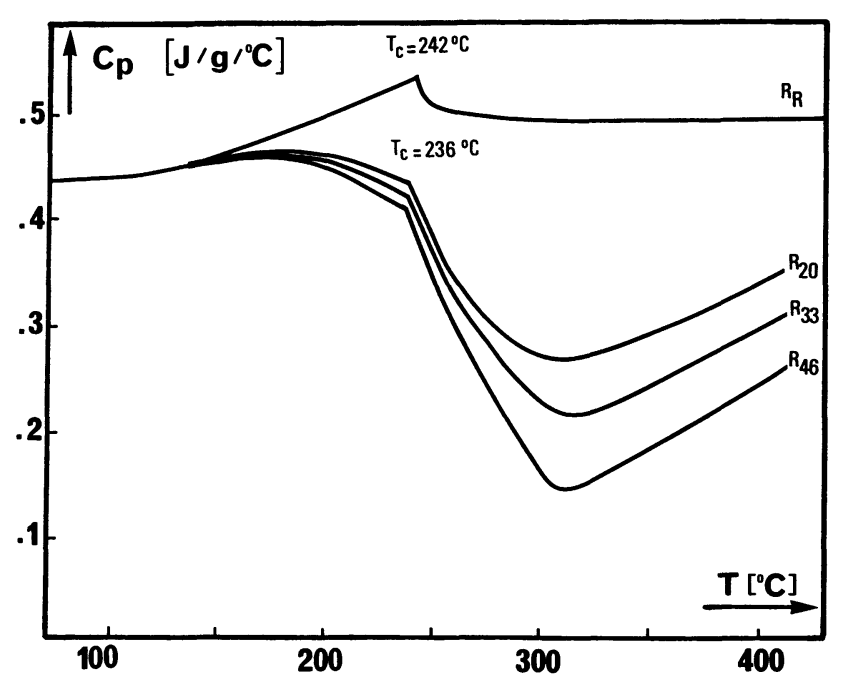

Fig. 4. - Variations de $C_{\mathrm{p}}$ en fonction de la température pour les rubans $\mathbf{R}_{20}, \mathbf{R}_{33}$ et $\mathbf{R}_{46}$ à l'état brut de trempe et pour ces mêmes rubans après relaxation structurale $\left(\mathbf{R}_{\mathrm{R}}\right)$.

$\left[C_{\mathrm{p}}\right.$ variations as a function of temperature for asquenched $R_{20}, R_{33}$ and $R_{46}$ ribbons and for the same ribbons after structural relaxation $\left(R_{R}\right)$.]

importante lors de la relaxation structurale. Pour autant, cette différence d'état structural semble sans effet sur la température de Curie $T_{\mathrm{C}}$; ce fait peut paraître surprenant quand on sait que $T_{\mathrm{C}}$ est très sensible à la relaxation structurale des verres métalliques $[10,11]$. Cette indépendance de $T_{\mathrm{C}}$ en fonction de la vitesse d'élaboration a été confirmé par une étude des propriétés magnétiques menée récemment par Le Gal et al. [15] sur les mêmes rubans.

\subsection{VARIATION DE $T_{\mathrm{C}}$ LORS D'UN RECUIT. - Nos} résultats précédents nous ont permis de mettre en évidence une augmentation de la température de Curie $T_{\mathrm{C}}$, lors de la relaxation structurale (cf. paragraphes 3.2 et 3.3) mais aucune variation sensible de $T_{\mathrm{C}}$ avec la vitesse d'hypertrempe (cf. paragraphe 3.4). Ces résultats sont apparemment contradictoires et nous avons étudié plus précisément l'influence de cette vitesse d'hypertrempe sur la relaxation structurale de l'alliage. Pour cela, des essais ont été effectués sur des échantillons prérecuits à l'extérieur du calorimètre. Deux types de traitements thermiques ont été réalisés ; traitements isochrones de $1 \mathrm{~h}$ à différentes températures comprises entre 100 et $425^{\circ} \mathrm{C}$ et traitements isothermes à 250 et $350^{\circ} \mathrm{C}$. Dans les deux cas nous avons suivi l'évolution des thermogrammes et plus particulièrement de $T_{\mathrm{C}}$ en fonction de la température ou du temps de recuit et ce, pour les rubans $R_{20}$ et $\mathbf{R}_{46}$ correspondant aux vitesses d'élaboration extrêmes. La figure 5 montre la variation de $T_{\mathrm{C}}$ en fonction de la température de recuit ; sans change-

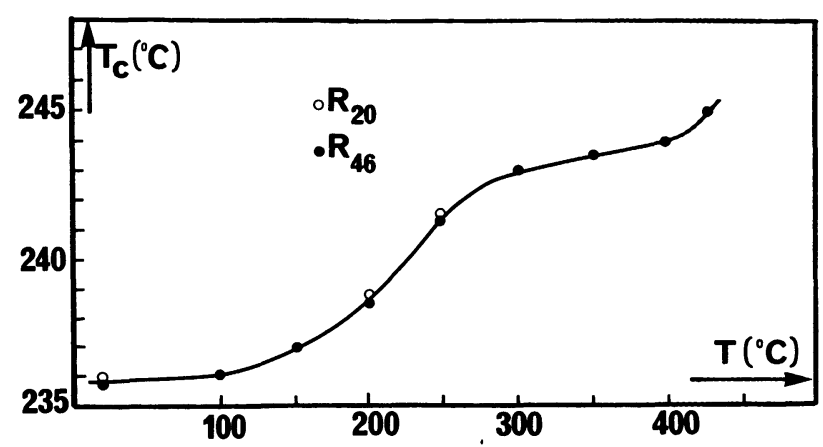

Fig. 5. - Variation de la température de Curie en fonction de la température de recuit (traitements isochrones de $1 \mathrm{~h}$ ) pour les rubans $R_{20}$ et $R_{46}$.

[Curie temperature variation as a function of annealing temperature (isochronal treatments during $1 \mathrm{~h}$ ) for $R_{20}$ and $\mathbf{R}_{46}$ ribbons.]

ment jusqu'à $100^{\circ} \mathrm{C}, T_{\mathrm{C}}$ subit ensuite une augmentation nette entre 150 et $300^{\circ} \mathrm{C}$, puis plus lente de 300 à $400^{\circ} \mathrm{C}$. Au-dessus de $400^{\circ} \mathrm{C}$, la cristallisation apparaît et à partir de $450^{\circ} \mathrm{C}$ il n'est plus possible de déterminer la température de Curie de la phase amorphe. De ces traitements isochrones (Fig. 5), il faut surtout retenir que $T_{\mathrm{C}}$ évolue de façon rigoureusement identique pour les deux rubans $R_{20}$ et $R_{46}$. La figure 6 montre à titre d'exemple comment évolue les chaleurs spécifiques apparentes d'échantillons du ruban $R_{20}$ après différents temps de traitement à $350^{\circ} \mathrm{C}$. L'effet exothermique associé à la relaxation structurale qui apparaît nettement audessus de $200^{\circ} \mathrm{C}$ pour l'état brut d'hypertrempe s'estompe rapidement et n'est plus visible pour les temps de recuit supérieurs à 3 min. Parallèlement un

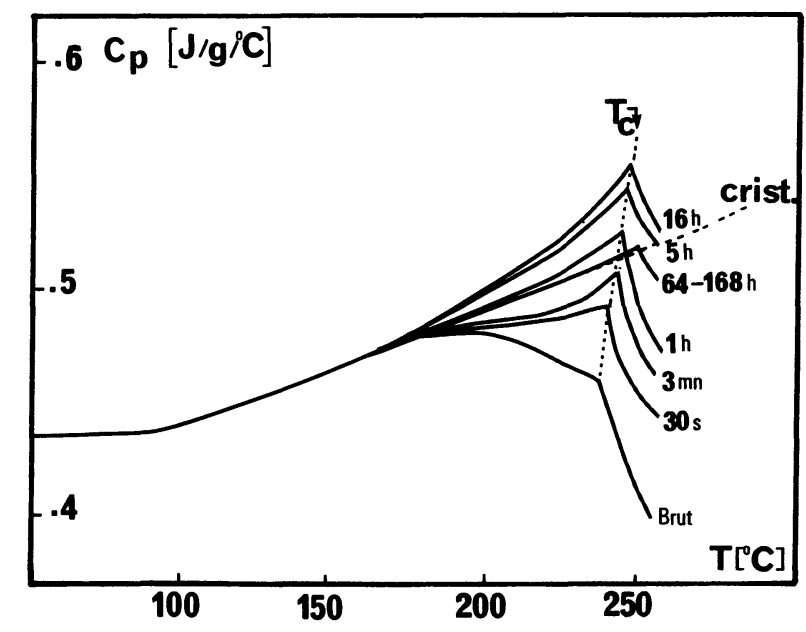

Fig. 6. - Evolution en fonction de la température de la chaleur spécifique apparente d'échantillons du ruban $R_{20}$ : Influence du temps de traitement à $350^{\circ} \mathrm{C}$.

[Apparent specific heat versus temperature for samples of the $R_{20}$ ribbon : Influence of the annealing time at $350{ }^{\circ} \mathrm{C}$.] 
effet endothermique centré sur $T_{\mathrm{C}}$ se développe pour atteindre un maximum pour un temps de recuit de $16 \mathrm{~h}$. Cet effet endothermique peut être associé bien sûr à la transition ferro-paramagnétique mais aussi à une variation réversible de l'ordre chimique à courte distance [11]. Après un temps de traitement de $64 \mathrm{~h}$ l'effet endothermique est considérablement réduit ; pour un tel traitement un début de cristallisation s'est produit, il ne subsiste plus que l'effet endothermique associé à la transition magnétique dans la phase amorphe restante. Nous pouvons enfin remarquer (Fig. 6) l'augmentation continue de la température de Curie avec le temps de recuit $\left(t_{\mathrm{R}}\right)$. Les variations de $T_{\mathrm{C}}$ en fonction de $t_{\mathrm{R}}$ ont été reportées sur la figure 7. Il faut tout d'abord noter

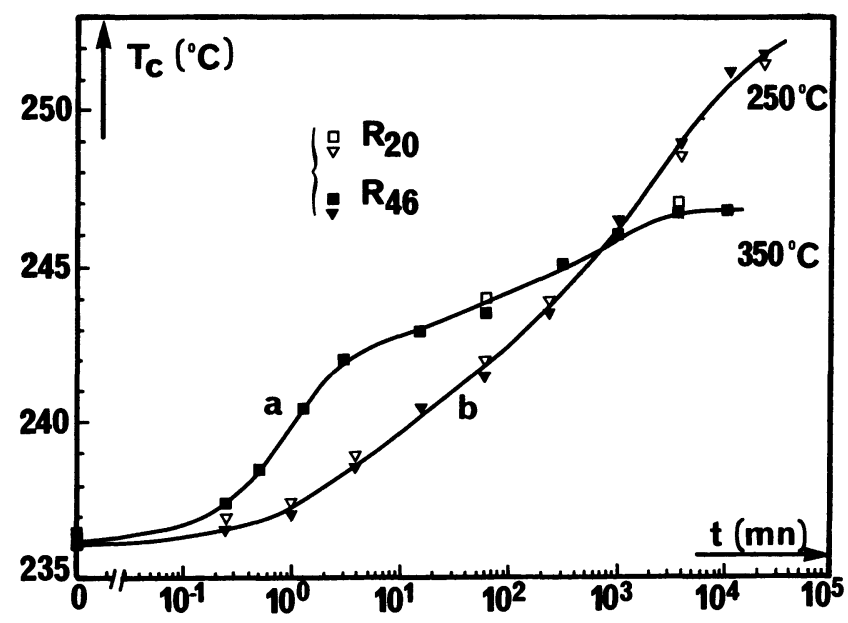

Fig. 7. - Variation de la température de Curie $T_{\mathrm{C}}$ en fonction du temps de recuit à $350^{\circ} \mathrm{C}$ (a) et $250^{\circ} \mathrm{C}$ (b) pour des rubans $\mathbf{R}_{20}$ et $\mathbf{R}_{46}$.

[Curie temperature variation versus annealing time at $350^{\circ} \mathrm{C} \mathrm{(a)}$ and $250^{\circ} \mathrm{C}(\mathrm{b})$ for $\mathrm{R}_{20}$ and $\mathrm{R}_{46}$ ribbons.]

que les cinétiques d'évolution de $T_{\mathrm{C}}$ sont les mêmes pour les rubans $R_{20}$ et $R_{46}$ que ce soit à $250^{\circ} \mathrm{C}$ ou à $350^{\circ} \mathrm{C}$. A $250^{\circ} \mathrm{C}, T_{\mathrm{C}}$ augmente progressivement de $236^{\circ} \mathrm{C}$ jusqu'à $252^{\circ} \mathrm{C}$, valeur à laquelle elle se stabilise après $160 \mathrm{~h}$ de traitement. A $350^{\circ} \mathrm{C}$, l'augmentation semble se produire en deux étapes: augmentation rapide jusqu'à $242^{\circ} \mathrm{C}$ après 3 min de recuit puis plus lente jusqu'à $246,5^{\circ} \mathrm{C}$, valeur atteinte après $64 \mathrm{~h}$ de recuit, temps pour lequel l'alliage a commencé à cristalliser. Pour un vieillisement à $350^{\circ} \mathrm{C}$, l'évolution de la température de Curie de la phase amorphe semblerait donc stoppée par l'apparition de la cristallisation.

Nous remarquons en outre (Fig. 7) que $T_{\mathrm{C}}$ se stabilise à deux valeurs très différentes pour les températures choisies. Après stabilisation à $250^{\circ} \mathrm{C}$, nous avons alors porté l'échantillon $4 \mathrm{~h}$ à $350^{\circ} \mathrm{C}$ et nous avons pu observer (Fig. 8) une diminution de $T_{\mathrm{C}}$ en dessous de sa valeur palier à $350^{\circ} \mathrm{C}$; une part

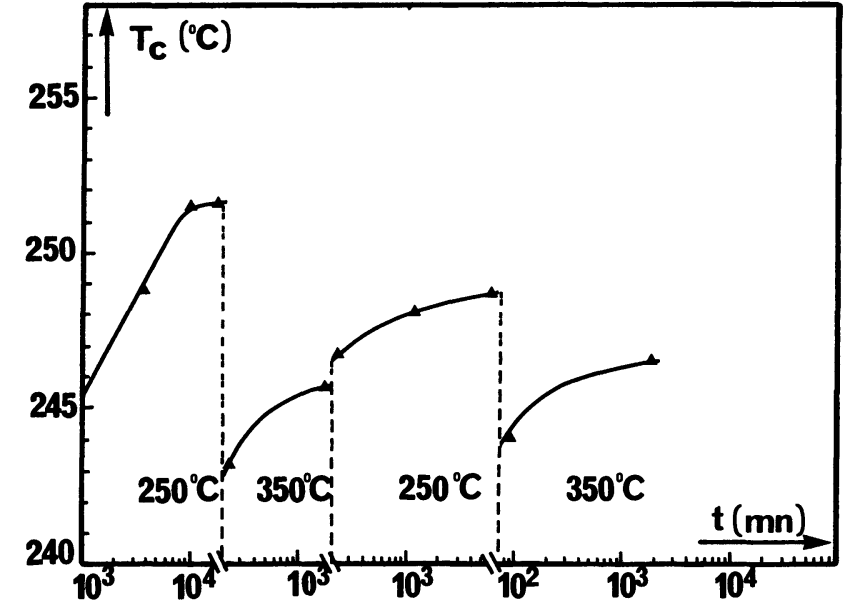

Fig. 8. - Evolution de $T_{\mathrm{C}}$ lors d'un cyclage thermique entre 250 et $350^{\circ} \mathrm{C}$.

[ $T_{\mathrm{C}}$ evolution during alternate heat treatments at 250 and $350^{\circ} \mathrm{C}$.]

importante de l'augmentation de $T_{\mathrm{C}}$ à $250^{\circ} \mathrm{C}$ serait donc associée à un changement réversible de l'ordre à courte distance dans la phase vitreuse ce que nous ont confirmé des cyclages thermiques entre 250 et $350^{\circ} \mathrm{C}$ (Fig. 8). Le fait, qu'au passage de 250 à $350^{\circ} \mathrm{C}, T_{\mathrm{C}}$ redescend en dessous de sa valeur palier à $350^{\circ} \mathrm{C}$ pour ensuite augmenter, au lieu de décroître purement et simplement peut être rapproché de ce que l'on peut observer dans des expériences dites de « cross-over » [16].

3.6 Discussion. - Nos résultats expérimentaux semblent montrer que l'incidence de la vitesse d'élaboration sur les températures des pics de cristallisation et sur la valeur ou les cinétiques d'évolution de la température de Curie apparaît comme étant négligeable. Le seul élément permettant de mettre en évidence une différence appréciable de cette vitesse sur l'état vitreux des rubans est la variation de l'effet exothermique associé à la relaxation structurale au cours d'un balayage en température (Fig. 4). Cette différence d'état structural ainsi décelée par l'analyse enthalpique différentielle étant insuffisante pour se traduire par une variation significative de la température de Curie, on est amené à conclure qu'elle doit être de faible importance. Pour faible qu'elle soit, cette différence entraîne pourtant des variations substantielles au niveau des propriétés mécaniques. Ainsi, comme nous l'avons montré par ailleurs $[12,13]$, le module d'Young et la fragilité des rubans augmentent fortement quand la vitesse d'élaboration diminue. Nos résultats nous ayant amené à conclure à l'absence de cristallisation à l'état brut d'hypertrempe (\$3.4), l'hypothèse d'une fragilisation due à l'existence d'une couche microcristalline en surface, comme l'ont proposé Janot et al. [17], ne semble pas pouvoir être retenue. Les faits 
expérimentaux tendraient donc à montrer qu'une faible modification de l'état vitreux lui-même peut se traduire par une importante variation de certaines propriétés (mécaniques), alors que d'autres sont peu affectées $\left(T_{\mathrm{C}}\right)$.

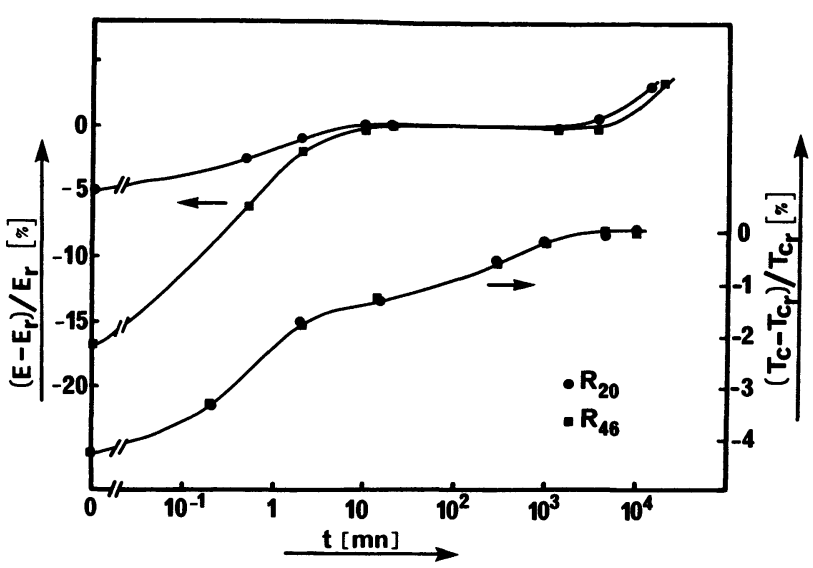

Fig. 9. - Variations relatives de $E$ et $T_{\mathrm{C}}$ au cours d'un recuit isotherme à $350^{\circ} \mathrm{C}$ pour les rubans $R_{20}$ et $R_{46}$ ( $E_{\mathrm{r}}$ et $T_{\mathrm{Cr}}$ module d'Young et température de Curie de l'alliage relaxé structuralement).

[Evolutions of $E$ and $T_{\mathrm{C}}$ during isothermal annealing at $350{ }^{\circ} \mathrm{C}$ for $\mathrm{R}_{20}$ and $\mathrm{R}_{46}$ ribbons $\left(E_{\mathrm{r}}\right.$ and $T_{\mathrm{Cr}}$ are the $E$ and $T_{\mathrm{C}}$ values of the relaxed alloys).]

La figure 9 compare à titre d'exemple les cinétiques d'évolution du module d'Young $E$ et de la température de Curie $T_{\mathrm{C}}$ à $350^{\circ} \mathrm{C}$ pour les rubans $\mathbf{R}_{20}$ et $\mathbf{R}_{46}$. Nous remarquons ainsi l'écart important sur la valeur de $E$ à l'état brut d'hypertrempe alors que $T_{\mathrm{C}}$ est identique dans les deux cas. Par ailleurs, le module atteint un palier après $10 \mathrm{~min}$ de recuit à $350^{\circ} \mathrm{C}$ alors que la température de Curie continue d'augmenter - la deuxième augmentation de $E$, après le palier, est associée à la cristallisation $-E$ et $T_{\mathrm{C}}$ ne semblent donc pas sensibles aux mêmes processus physiques ce qui montre que plusieurs mécanismes entrent en jeu lors de la relaxation structurale. Le mécanisme à l'origine physique de l'augmentation du module se produirait très rapidement, pendant l'élaboration même en ce qui concerne le ruban $R_{20}$, et n'aurait qu'une faible incidence sur $T_{\mathrm{C}}$. Le mécanisme à l'origine de l'augmentation de $T_{\mathrm{C}}$ n'aurait lui qu'une influence secondaire sur le module d'Young.

Pour expliquer ce comportement, il nous a paru intéressant d'utiliser la notion de défauts topologiques dans les amorphes introduites par Egami et al. [18-21] et reprise ensuite par divers auteurs pour interpréter les évolutions des propriétés physiques ou mécaniques [6, 22-24]. La définition de ces défauts repose sur l'évaluation des deux invariants du tenseur des contraintes atomiques que sont la pression hydrostatique et la contrainte de cisaille- ment de Von Misès. Les défauts sont ainsi définis comme des régions dans lesquelles l'un au moins des invariants du tenseur diffère de façon significative de sa valeur moyenne. On distingue trois types de défauts ; les défauts $p$ où la pression hydrostatique est supérieure à la moyenne et la densité atomique forte, les défauts $\mathbf{n}$ où la situation est opposée et les défauts $\tau$ où les contraintes de cisaillement sont importantes. Dans tous les cas, ces défauts topologiques sont des sites où les réarrangements atomiques sont susceptibles de se produire beaucoup plus rapidement que dans le reste de la matrice. La densité globale de défauts et les proportions relatives de chacun d'eux vont dépendre de la vitesse d'hypertrempe et vont évoluer au cours de la relaxation structurale qui peut être pour une grande part décrite comme une conséquence de l'annihilation des défauts [20] s'accompagnant d'une mise en ordre à courte distance.

Pour interpréter la différence d'évolution de la température de Curie et du module d'Young avec la vitesse d'hypertrempe et au cours de la relaxation structurale, nous pouvons alors envisager les deux hypothèses suivantes :

i) le module d'Young est surtout sensible à la présence des défauts topologiques. Les réarrangements atomiques étant particulièrement rapides au niveau de ces sites, un ordre à courte distance peut facilement s'y créer dans les premiers temps de la relaxation structurale, voire pendant le refroidissement en dessous de $T_{\mathrm{g}}$ lors de l'élaboration. Un certain nombre de ces défauts serait ainsi neutralisé et leur efficacité vis-à-vis du module d'Young diminuée; l'augmentation du module pourrait être le résultat soit d'une modification du potentiel d'interaction moyen, soit de l'annihilation d'une anomalie de module associée à la grande facilité des déplacements atomiques au niveau des défauts. Un tel mécanisme permet d'expliquer d'une part la différence de module d'Young entre les rubans $R_{20}$ et $\mathbf{R}_{46}$ à l'état brut d'hypertrempe et d'autre part, la diminution de l'effet exothermique associé à la relaxation structurale quand on passe des rubans $\mathbf{R}_{\mathbf{4 6}}$ à $\mathbf{R}_{\mathbf{2 0}}$. La stabilisation du module après $10 \mathrm{~min}$ de recuit à $350^{\circ} \mathrm{C}$ signifierait que l'on a éliminé ou neutralisé la quasi-totalité des défauts hors d'équilibre et atteint ainsi la fin du premier stade de la relaxation structurale. La densité des défauts restant ensuite constante, le module ne varie plus sensiblement. Ce mécanisme de création d'un ordre à courte distance sur les défauts serait de caractère essentiellement irréversible et permettrait en outre d'expliquer la fragilisation de l'alliage, l'unité structurale à mouvoir pour permettre le cisaillement localisé à l'origine de la déformation non élastique dans les verres métalliques [23] n'étant plus un atome seul mais par exemple un atome métallique entouré de métalloïdes. Ce problème de la fragilisation en 
liaison avec la vitesse d'hypertrempe et la relaxation structurale a été étudié de façon détaillée par ailleurs $[12,13]$.

ii) la température de Curie dépend pour l'essentiel de l'environnement atomique des porteurs de moment magnétique, c'est-à-dire de l'ordre à courte distance. La création de cet ordre chimique à courte distance est, dans le premier stade de la relaxation structurale facilitée par l'annihilation des défauts qui contribue à la diffusion dans la matrice amorphe et l'on observe une variation rapide de $T_{\mathrm{C}}$. Quand les défauts hors d'équilibre à la température de recuit ont été éliminés, il se produit une redistribution des défauts à l'équilibre entraînant des réarrangements atomiques d'ordre chimique ou topologique qui s'étendent à l'ensemble de la matrice amorphe et conduisent à la lente augmentation de $T_{\mathrm{C}}$ observée après $10 \mathrm{~min}$ de recuit à $3500^{\circ} \mathrm{C}$.

Il reste néanmoins à expliquer l'effet négligeable de la vitesse d'élaboration sur la valeur et sur les cinétiques d'évolution de $T_{\mathrm{C}}$. Il nous faut alors admettre que, d'une part les réarrangements atomiques au cours de l'hypertrempe localisés au niveau des défauts, n'impliquent qu'un faible pourcentage des porteurs de moments magnétiques et que d'autre part l'ordre chimique dans le reste de la matrice amorphe dépend peu de la vitesse d'élaboration dans le domaine de vitesses étudié ; en d'autres termes, en dehors des défauts, les vitesses de refroidissement seraient toutes suffisamment élevées pour permettre de figer le désordre chimique existant à l'état liquide.

Par ailleurs, l'ordre qui s'établit au cours de la relaxation structurale, apparaît comme partiellement réversible. Cela signifierait que l'on a atteint l'équilibre métastable et que l'on peut faire évoluer réversiblement la structure vitreuse en fonction de la température. Il est ainsi possible d'envisager des fluctuations de l'arrangement des métallö̈des et du chrome autour du porteur de moment magnétique que constitue l'élément fer, conduisant à une variation réversible de $T_{\mathrm{C}}$. La densité de défauts à l'équilibre étant peu affectée au cours de ces processus de changement d'ordre avec la température, le module d'Young ne présentera pas une telle réversibilité. Des variations réversibles du module élastique ont pu être observées sur certains verres métalliques $[25,26]$ mais cette composante réversible reste toujours très faible comparée à l'augmentation globale du module associée à la relaxation structurale.

\section{Conclusion.}

Dans le domaine des vitesses d'hypertrempe exploré, aucune influence de cette vitesse n'a été décelée visà-vis de la cristallisation du verre métallique, que ce soit au niveau des températures, de la variation d'enthalpie ou des énergies d'activation apparentes correspondant aux deux stades de cristallisation.

L'analyse enthalpimétrique ne montre une différence qu'au niveau de l'effet exothermique associé à la relaxation structurale du verre : cet effet est d'autant plus marqué que la vitesse d'hypertrempe est grande, ce qui traduit le fait que le verre est dans un état d'autant plus proche de l'équilibre métastable que cette vitesse est faible. Cette différence n'est néanmoins pas suffisante pour induire une variation de la température de Curie, grandeur pourtant très sensible à la relaxation structurale des verres métalliques ferromagnétiques. Par contre, pour ces mêmes rubans, des variations substantielles de certaines propriétés comme le module d'Young et la fragilité ont pu être observées $[12,13]$. La comparaison des évolutions de la température de Curie et du module d'Young au cours de la relaxation structurale, ont permis de conclure que les variations de ces deux grandeurs n'ont pas les mêmes origines physiques.

A partir de la notion de défauts dans les amorphes métalliques et de la théorie de la relaxation structurale basée sur l'existence de ces défauts développée par Egami et al. [18-21] il nous a été possible d'interpréter nos résultats en décomposant cette relaxation structurale en deux grands stades :

i) «neutralisation » et/ou annihilation des défauts topologiques hors d'équilibre. Du fait de la grande facilité des réarrangements atomiques au niveau des défauts le processus de "neutralisation » de ces sites par création d'un ordre local peut débuter au cours même du refroidissement en dessous de $T_{\mathrm{g}}$ lors de l'hypertrempe. Ce processus permet alors d'expliquer l'augmentation du module et de la fragilité et la variation négligeable de la température de Curie quand la vitesse d'élaboration diminue.

ii) redistribution des défauts à l'équilibre s'accompagnant d'une mise en ordre à courte distance dans l'ensemble de la matrice amorphe qui se traduit par une augmentation de $T_{\mathrm{C}}$, le module d'Young restant constant.

Par ailleurs, l'ordre chimique à courte distance, créé parallèlement à l'annihilation des défauts hors d'équilibre ou à la redistribution des défauts à l'équilibre, présente un caractère partiellement réversible. La température de Curie apparaît sensible à cette variation réversible de l'ordre alors que le module d'Young - fonction essentiellement de la densité des défauts $-\mathrm{y}$ est insensible.

\section{Remerciements.}

Cette étude a été réalisée grâce à une participation financière du Centre de Recherches de Pont-àMousson. 


\section{Bibliographie}

[1] Takayama, S., Oı, T., J. Appl. Phys. 50, (1979) 1595.

[2] Luborsky, F. E., LiebermanN, H. H., Walter, J. L., Metallic Glasses, Sci. Techn., Budapest 1 (1981) 203.

[3] Mizoguchi, T., Hatta, S., Kato, H., Arai, H., MAedA, K., AKuTsu, N., I.E.E.E. Trans. Magn. Mag. 16 (1980) 1147.

[4] Vojtanik, P., Ротоcky, L., Boskovi-Cova, M., Kisdi-Koszo, E., Lovas, A., Acta Phys. Slov. 31 (1981) 109.

[5] Greer, A. L., J. Mat. Sci. 17 (1982) 1117.

[6] Gerling, R., Wagner, R., Scripta Met. 17 (1983) 1129.

[7] Chen, H. S., Krause, J. T., Inoue, A., MasuMOTO, T., Scripta Met. 17 (1983) 1413.

[8] Koster, U., Scripta Met. 17, (1983) 867.

[9] Grossinger, R., SASsiK, H., Proceedings of Rapidly Quenched Metals V, vol. 2, 1255, (1984).

[10] Davies, H. A., Gregan, G. P. J., J. Non Cryst. Solids 61-62 (1984) 823.

[11] Zielinski, P. G., Ast, D. G., J. Non Cryst. Solids 61-62 (1984) 1021.

[12] Allemand, J. P., Thèse de doctorat, I.N.S.A. Lyon (1986).
[13] Allemand, J. P., Fouguet, F., Perez, J., De GuILlEBON, B., à paraître.

[14] Boswell, P. G., J. Thermal Analysis 18, 353 (1980).

[15] Le Gal, G., Henry, M., VARret, F., à paraître.

[16] Greer, A. L., Leake, J. A., J. Non Cryst. Solids 33, 291 (1979).

[17] Janot, C., George, B., Marchal, G., Delcroix, P., J. Physique Colloq. 43 (1982) C9-209.

[18] Egami, T., Maeda, K., Vitek, V., Philos. Mag. 41 (1980) 883.

[19] Egami, T., Vitek, V., Srolovitz, D., Proceedings of Rapidly Quenched Metals IV, vol. 1 (1981) p. 517.

[20] Srolovitz, D., Maeda, K., Vitek, V., Egami, T., Philos. Mag. 44 (1981) 847.

[21] Srolovitz, D., Vitek, V., EgAmi, T., Acta Met. 31 (1983) 335.

[22] Perez, J., Fouquet, F., Lormand, G., Les Amorphes Métalliques, Ecole d'hiver d'Aussois (Les Editions de Physique) 1983, p. 537.

[23] Perez, J., Acta Met. 32 (1984) 2163.

[24] Morito, N., EgAmi, T., Acta Met. 32 (1984) 603.

[25] ScotT, M. G., Kursumovic, A., Met. Sci. 15 (1981) 583.

[26] Balanzat, E., Thèse de doctorat d'état, Grenoble (1983). 